\title{
DIAGNÓSTICOS REGIONALES SOBRE LA INSTITUCIONALIZACIÓN DEL COMPROMISO AMBIENTAL EN LA EDUCACIÓN SUPERIOR DE AMÉRICA LATINA Y EL CARIBE
}

\author{
DIAGNÓSTICOS REGIONAIS SOBRE A INSTITUCIONALIZAÇÃO DO COMPROMISSO AM- \\ BIENTAL NO ENSINO SUPERIOR DA AMÉRICA LATINA E DO CARIBE \\ REGIONAL DIAGNOSES OF THE INSTITUTIONALIZATION OF ENVIRONMENTAL COM- \\ MITMENT IN LATIN AMERICAN AND CARIBBEAN HIGHER EDUCATION
}

Orlando Sáenz ${ }^{1}$

${ }^{1}$ Docente del Programa de Maestría en Ciencias Ambientales de la Universidad de Ciencias Aplicadas y Ambientales (UDCA) - Bogotá - Colombia.

Resumen: En América Latina y el Caribe se cuenta con varias decenas de evaluaciones, informes, diagnósticos, reportes, inventarios, auditorías y otros trabajos similares sobre la ambientalización de las instituciones de educación superior (IES). Unos pocos de ellos son estudios de escala regional o subregional. En la primera parte de este artículo se reseñan los diagnósticos regionales realizados entre los años setenta y comienzos de este siglo, así como los más recientes desarrollados durante el decenio en curso. Solo los últimos estudios intentan articularse para facilitar el conocimiento de los avances de las universidades en dicho proceso. En el tercer apartado se presenta un trabajo que se adelanta desde 2014 para producir una serie de diagnósticos nacionales con indicadores comunes que permitirán tener a mediano plazo un gran diagnóstico regional. A continuación, se hace un balance general de los estudios adelantados durante cuatro décadas para conocer el proceso por el cual las IES de América Latina y el Caribe han venido asumiendo gradualmente su compromiso institucional con el ambiente y la sustentabilidad. Finalmente, se recuerda la Agenda para la Sostenibilidad en las Universidades con sus tres componentes y se propone, como un instrumento integrador, la construcción de un Observatorio de la Sustentabilidad en la Educación Superior (OSES) de la región.

Palabras clave: Sustentabilidad en las universidades; Incorporación de la dimensión ambiental en la educación superior; Universidad y ambiente.

Resumo: Na América Latina e no Caribe existem dezenas de avaliações, relatórios, diagnósticos, inventários, auditorias e outras obras semelhantes sobre a ambientalização das instituições de ensino superior (IES). Apenas alguns deles são estudos de âmbito regional ou sub-regional. Na primeira parte deste artigo são descritos os diagnósticos regionais realizados entre a década de 1970 e início deste século, bem como os mais recentes desenvolvidos durante o decênio atual. Só os estudos mais recentes estão tentando articular-se para facilitar o conhecimento dos progressos das universidades neste processo. A terceira seção apresenta um trabalho que se desenvolve desde 2014 para produzir uma série de diagnósticos nacionais com indicadores comuns que permitiram ter a médio prazo um grande diagnóstico regional. Segue uma avaliação geral dos estudos desenvolvidos ao longo de quatro décadas para conhecer o processo pelo qual as IES da América Latina e do Caribe têm assumido gradualmente seu compromisso institucional com o meio ambiente e a sustentabilidade. Finalmente, 
lembra-se da Agenda para a Sustentabilidade nas Universidades com seus três componentes e se propõe, como um instrumento integrador, a construção de um Observatório da Sustentabilidade no Ensino Superior (OSES) na região.

Palavras-chave: Sustentabilidade nas universidades; Incorporação da dimensão ambiental no ensino superior; Universidade e meio ambiente.

\begin{abstract}
In Latin America and the Caribbean, there are dozens of evaluations, reports, diagnoses, inventories, audits and other similar works on the "environmentalization" of higher education institutions (HEIs). Of these, only some are regional or subregional studies. The first part of this article brings the regional diagnoses carried out between the 1970s and the beginning of this century, as well as the most recent studies developed during the current decade. Only the most recent studies bring an attempt to facilitate knowledge of the evolution of universities in this process. The third section presents a study that has been developed since 2014 aiming to produce a series of national diagnoses using common indicators, which will allow a large medium-term regional diagnosis. Next, it presents the general evaluation of the studies developed over the four decades to unveil the process by which HEIs in Latin America and the Caribbean have gradually committed to the environment and sustainability guidelines. Finally, it brings a recall of the Agenda for Sustainability in universities, with its three components, to then propose, as an integrating tool, the construction of an Observatory of Sustainability of Higher Education (OSES) in the region.
\end{abstract}

Keywords: Sustainability in universities; Inclusion of the environmental dimension into higher education; University and the environment.

\title{
INTRODUCCIÓN
}

Desde mediados de la década del setenta, en el siglo pasado, se vienen desarrollando una serie de evaluaciones, informes, diagnósticos, reportes, inventarios y otros trabajos similares sobre el proceso que en un principio se denominó "incorporación de la dimensión ambiental en la educación superior". En el primer número de la Revista AMBIENS se publicó el artículo Diagnósticos Nacionales sobre la Inclusión de Consideraciones Ambientales en las Universidades de América Latina y El Caribe (SÁENZ, 2015b). Allí se enumeran y describen brevemente un total de 40 estudios nacionales, desarrollados en 11 países de la región.

En los dos primeros apartados de este artículo se presentan los resultados de una revisión que pretende ser exhaustiva de los principales diagnósticos de escala regional sobre la ambientalización de las instituciones de educación superior en América Latina y el Caribe. Son mucho menos numerosos que los diagnósticos nacionales y se pueden dividir en dos grupos: a) Los primeros trabajos de este tipo realizados entre los años setenta y comienzos de este siglo; b) Los estudios más recientes desarrollados durante el último decenio.

La gran mayoría de los diagnósticos que se tienen hasta ahora han estado desarticulados. Casi todos se presentan como trabajos aislados, sin referencia a los que le precedieron. Mucho menos ha habido un intento de actualizar la información de estudios previos. Por lo tanto, con base en ellos no es posible hacer un seguimiento confiable de los avances en el proceso de institucionalización del compromiso ambiental en las universidades latinoamericanas y caribeñas. 
Para tener este conocimiento, desde 2014 se viene desarrollando un trabajo que intenta llegar a un diagnóstico regional de este proceso a partir de una serie de diagnósticos nacionales, con base en un grupo de indicadores comunes, para permitir la comparabilidad entre países y la agregación de datos obtenidos en las respectivas encuestas. En el tercer apartado se presenta el origen y los avances de esta investigación colaborativa internacional desarrollada en el marco de la Alianza de Redes Iberoamericanas de Universidades por la Sustentabilidad y el Ambiente (ARIUSA).

En el siguiente apartado de este artículo se hace un balance general del trabajo desarrollado durante cuatro décadas para conocer el proceso por el cual las universidades de América Latina y el Caribe han venido asumiendo gradualmente su compromiso institucional con el ambiente y la sustentabilidad. Allí se señalan algunas de las deficiencias metodológicas de los diagnósticos regionales y la imposibilidad de construir a partir de ellos una imagen coherente de las diferentes etapas de dicho proceso y de los avances que se han logrado.

Para finalizar, se recuerda la Agenda para la Sostenibilidad en las Universidades planteada por la Alianza de Redes Iberoamericanas de Universidades por la Sustentabilidad y el Ambiente (ARIUSA). Después de analizar el cumplimiento de los tres objetivos o componentes de esta agenda, se propone, como un instrumento integrador, la construcción de un Observatorio de la Sustentabilidad en la Educación Superior (OSES) de la región.

\section{Primeiros diagnósticos REgIONALES}

El primer diagnóstico sobre los avances en la incorporación de la dimensión ambiental en las universidades latinoamericanas fue realizado por el Centro Internacional de Formación en Ciencias Ambientales (CIFCA). Este centro fue creado en 1975 como resultado de un acuerdo entre el Programa de las Naciones Unidas para el Medio Ambiente (PNUMA) y el Gobierno de España. Desde un comienzo orientó su trabajo hacia la promoción de la educación ambiental a nivel superior en los países de habla castellana.

El CIFCA realizó en 1977 el estudio titulado Panorama de los Estudios Superiores Medioambientales en América Latina, para el que se recopiló información de 12 países latinoamericanos de lengua española (Ver Tabla No. 2). Aunque con importantes limitaciones desde el punto de vista metodológico, este primer diagnóstico regional logró identificar una serie de "tendencias generales en la incorporación del tema del medio ambiente en la educación superior" de América Latina y el Caribe (CIFCA, 1978, p.57).

En el nivel de pregrado se identificaron las primeras formas de introducir los temas ambientales en los programas de formación universitaria latinoamericana: a) La ampliación del contenido curricular con nuevas asignaturas o cursos relacionados con los temas ambientales; y b) La "creación de programas experimentales vinculados más directamente con los temas concretos de la dimensión ambiental". A nivel de postgrado se identificó "una marcada tendencia hacia la especialización de los profesionales", así como el surgimiento de nuevos 
"programas en torno a áreas problema en que se aborda un tema común desde la perspectiva de diferentes disciplinas" (CIFCA, 1978, p.67).

Los funcionarios del CIFCA reconocían "con toda honestidad las limitaciones del esfuerzo realizado, cuyos resultados son todavía preliminares, además de incompletos". Sin embargo, afirmaban que se trataba apenas de "un primer avance de la investigación de más largo aliento" (CIFCA, 1978, p.57) que planeaban realizar en los años siguientes con el apoyo de la emergente comunidad universitaria ambientalista de Iberoamérica. Desafortunadamente, esta investigación nunca logró terminarse.

Después del cierre del CIFCA, a comienzos de la década del ochenta, el trabajo de diagnóstico y promoción de la formación ambiental superior en la región fue continuado por la Oficina Regional para América Latina y el Caribe (ORPALC) del Programa de las Naciones Unidas para el Medio Ambiente (PNUMA). Esta labor se intensificó con la creación de la Unidad de Coordinación (UCORED) y del programa Red de Formación Ambiental para América Latina y el Caribe (RFA-LAC), en 1981 y 1982 respectivamente, los cuales trabajaron en estrecha cooperación con el CIFCA durante sus primeros dos años.

Después de creada la RFA-ALC, una de las primeras tareas que asumió la UCORED fue la evaluación del nivel de desarrollo alcanzado por la formación ambiental superior en la región. Con este propósito, en 1984 se adelantó el Diagnóstico de la Incorporación de la Dimensión Ambiental en los Estudios Superiores en América Latina y el Caribe, en el que se obtuvo información sobre 166 universidades de 18 países latinoamericanos (Ver Tabla No. 2), "lo que representaba casi el 50\% de las universidades de la región" (UNESCO, PNUMA \& ICFES, 1988, p.20). Los resultados de este estudio regional se presentaron en el primer Seminario sobre Universidad y Medio Ambiente en América Latina y el Caribe, que se realizó en Bogotá (Colombia) a finales de 1985.

Entre los resultados de este diagnóstico del PNUNA se puede destacar que "en total se identificaron 121 carreras, de las cuales 31 estaban en fase de proyecto" (UNESCO, PNUMA \& ICFES, 1988, p.24). La mayor parte de estas carreras se daban en el campo de la Ecología, de las Ingenierías, de las Ciencias de la Salud y de las Ciencias Ambientales. También se encontró que en algunas universidades latinoamericanas y caribeñas comenzaban a modificarse los objetivos y los contenidos de carreras y de cátedras tradicionales para asumir la nueva perspectiva ambiental. Así, "19 carreras informaron haber transformado objetivos y contenidos para acoplarlas a la problemática ambiental y 18 cátedras aparecen transformando sus contenidos" con el mismo propósito (UNESCO, PNUMA \& ICFES, 1988, p.28).

En general, el estudio del PNUMA permitió constatar que, a mediados de la década del ochenta, las universidades de América Latina y el Caribe ya estaban desarrollando múltiples actividades ambientales en sus programas de docencia, investigación y extensión (UNESCO, PNUMA \& ICFES, 1988, pp.20 a 26), pero el proceso de incorporación de la dimensión ambiental en la educación superior de la región todavía enfrentaba serios obstáculos para su consolidación. Varios de ellos derivaban de la tradicional estructura académica que con dificultades responde a las exigencias de cambios en el enfoque epistemológico y en el modelo organizacional de las IES, planteadas por la incorporación de la dimensión ambiental. 
Este trabajo del PNUMA en 1984 "constituyó el mayor esfuerzo realizado a nivel regional para diagnosticar el grado de avance de los programas ambientales en las universidades de la región, así como para intercambiar experiencias y discutir conceptos, orientaciones y criterios sobre la incorporación de la dimensión ambiental en las prácticas académicas y de investigación" (UNESCO, PNUMA \& ICFES, 1988, p.9). Hasta hoy en día, continúa siendo el único diagnóstico, con una amplia base de información empírica y cobertura regional, que se tiene en América Latina y el Caribe sobre los programas ambientales universitarios.

Desde comienzos de la década del noventa se viene desarrollando la serie de Congresos Iberoamericanos de Educación Ambiental (CIEA) como un espacio de reunión periódica de la comunidad de educadores ambientales en el que, de hecho y entre otras actividades, se presentan ponencias e informes que permiten hacer un seguimiento, más o menos cercano, del rumbo que ha tomado el proceso de ambientalización de la educación superior en los países ibéricos y latinoamericanos durante los últimos decenios. Así ocurrió con el evento inaugural de la serie, que tuvo lugar en Guadalajara (México) en noviembre de 1992. En el libro de memorias, titulado Educación Ambiental y Universidad (CURIEL, 1993), se recopilaron las conferencias centrales del primer Congreso y casi 30 trabajos cuyos temas se referían a distintos aspectos de la educación ambiental superior.

Uno de esos trabajos fue precisamente la ponencia del Coordinador de la RFA-ALC en la que intentó "rescatar algunos de los hitos de este proceso y hacer un balance de logros" (LEFF, 1993, p.75) hasta ese momento. Como parte de este balance, reconocía que "a pesar de los esfuerzos realizados, la puesta en marcha de programas ambientales en la región ha sido relativamente lenta, lo que se traduce en la falta de profesionales capacitados para la elaboración y ejecución de políticas ambientales". En el mismo sentido, se planteaba que, "aunque se ha avanzado en el desarrollo del saber ambiental en áreas temáticas, no ha habido un esfuerzo igual para incorporarlo en nuevos contenidos curriculares y poco se ha avanzado en estructurar programas interdisciplinarios de docencia e investigación en temas ambientales". En general, se afirmaba que "es evidente que estamos lejos de haber consolidado la profesionalización de la formación ambiental" en "un proceso que ha estado caracterizado por su falta de continuidad, con una serie de avances y retrocesos" (LEFF, 1993, pp. 77 y 79).

En el 2000 se celebró el III Congreso Iberoamericano de Educación Ambiental en Caracas (Venezuela). En este tercer CIEA se realizó un esfuerzo importante para elaborar "un diagnóstico de la situación de la educación ambiental en la región". Con este propósito, se prepararon una serie de "informes diagnósticos del estado de la educación ambiental en cada uno de los países de la región" que se presentaron en el I Simposio de Países Iberoamericanos sobre Políticas y Estrategias Nacionales de Educación Ambiental, reunido en el marco del Congreso. En el Simposio se presentaron un total de 13 informes nacionales, cada uno de ellos preparado para "describir de manera abreviada el estado del arte del campo educativo ambiental en cada uno de esos países, discutir posibles tendencias existentes y en proponer posibles orientaciones para la gestión de estos programas educativos" (ÁLVAREZ et al, 2002, p.645).

En 10 de estos informes nacionales se incluyó un apartado dedicado específicamente a la educación ambiental superior (Ver Tabla No. 2). En la mayoría de ellos se presentó una breve 
reseña histórica y algunos análisis del proceso de incorporación de la dimensión ambiental en las universidades. Entre las conclusiones del Simposio se destaca el reconocimiento de "un avance de la educación ambiental en el ámbito de la educación superior. En este sentido se observa un aumento en las carreras de pre y postgrado en el campo ambiental en general y en el de la EA en particular" (ÁLVAREZ et al, 2002, p. 651).

Un poco después, el PNUMA intentó construir un Directorio de Instituciones y Programas de Formación Ambiental en América Latina y el Caribe. Con este propósito, la Coordinación del Programa RFA-ALC adelantó una encuesta entre 2003 y 2004. Sin embargo, la recopilación de información se limitó a los programas de postgrado y el nivel de respuesta útil al formulario que se utilizó fue menor al 15\%. "Se escribió a 432 universidades de 21 países latinoamericanos, de las cuales respondieron 80 " $\mathrm{y}$, de estas respuestas, " 16 formularios son inservibles ya que están incompletos" (ESCHENHAGEN, 2009, pp.173 y 174). Las respuestas recibidas correspondían a universidades de 13 países (Ver Tabla No. 2),

Los datos obtenidos fueron luego utilizados en una investigación de Doctorado para analizar la Oferta de Programas de Posgrado en América Latina. A pesar de las limitaciones de la información, se pudieron reconocer algunas tendencias importantes, "como la falta de doctorados, la débil presencia de las Ciencias Sociales, la insuficiente presencia de la interdisciplinariedad, la ausencia completa de educación ambiental en muchos países pequeños de América Latina y el Caribe y, a la vez, un aumento considerable de la oferta de programas" (ESCHENHAGEN, 2009, p.95).

\section{DiAgnósticos REgIONALES RECIENTES}

Durante el segundo decenio del siglo XXI ha aumentado el interés por conocer los avances en el proceso de ambientalización de las instituciones de educación superior en América Latina y el Caribe. De hecho, en unos pocos años se tiene un número de diagnósticos regionales casi igual a los que adelantaron durante las cuatro décadas anteriores en la región.

El único estudio de escala subregional que se conoce sobre la ambientalización de las universidades en Latinoamérica es el Report of the Audit - Mainstreaming Environment and Sustainability in Caribbean Universities (UWI \& UNEP, 2011). Este estudio fue coordinado, durante 2010, por la red Mainstreaming of Environment and Sustainability in Caribbean Universities (MESCA) y financiado por la oficina central en Nairobi (Kenia) del United Nations Environment Programme (UNEP). Para la ejecución de esta auditoría ambiental se adaptó un instrumento desarrollado por la red Mainstreaming Environment and Sustainability in African Universities (MESA). Participaron 6 universidades de tres países caribeños y uno centroamericano de habla inglesa (Ver Tabla No. 2).

El principal objetivo de este ejercicio de auditoría ambiental a instituciones de educación superior en el Caribe fue "identificar en qué medida los temas, las preocupaciones y las prácticas de sostenibilidad son parte de los programas y operaciones de las universidades" (UWI \& UNEP, 2011, p. 5). Sus resultados no fueron muy claros debido a la heterogeneidad de formas en que las universidades reportaron su información. De manera general, se encontró 
que el área de formación ambiental fue la mejor evaluada por "la capacidad y la voluntad del personal docente para las actividades relacionadas con la sostenibilidad", que se expresa en la introducción de este concepto en algunos de los cursos (NAVARRO, 2015, p. 252). Por otra parte, aunque el paisajismo sostenible es una práctica común entre las universidades participantes en dicho estudio, la gestión ambiental, relacionada con desechos, energía y agua presentaba un bajo desarrollo (UWI \& UNEP, 2011, p. 5).

Obviamente, el interés por conocer el nivel de avance del proceso de ambientalización de las instituciones de educación superior no se limita a América Latina y el Caribe. También se encuentra en otras regiones del mundo y a nivel global.

El primer estudio a escala mundial se presentó en 2012 en el libro Higher Education in the World 4. Higher Education's Commitment to Sustainability: from Understanding to Action. Fue el cuarto volumen de la serie de informes mundiales sobre diferentes aspectos de la educación superior que publica la Global University Network for Innovation (GUNi). En este reporte se presenta una visión general sobre el compromiso de las universidades con la sustentabilidad, a través de seis informes regionales (LOTZ-SISITKA, 2012; SALAMÉ, 2012; KO \& ABE, 2012; RIKERS \& de SNOO \& van DAM-MIERAS, 2012, WRIGTH \& ELLIOT 2012, SÁENZ \& BENAYAS, 2012). Adicionalmente, analiza las tendencias, redes, experiencias y buenas prácticas de las instituciones de educación superior en el mundo en materia de ambiente y sustentabilidad (GUNi, 2012).

El reporte mundial de GUNi incluyó el artículo Higher Education, Environment and Sustainability in Latin America and the Caribbean (SÁENZ \& BENAYAS, 2012), en el que se presenta una descripción general del proceso histórico y la situación actual de la incorporación de la dimensión ambiental en las instituciones de educación superior en la región. La versión en español de este artículo se publicó en el segundo número de la Revista AMBIENS, con el título Ambiente y Sustentabilidad en las Instituciones de Educación Superior en América Latina y el Caribe (SÁENZ \& BENAYAS, 2015). Desde una perspectiva histórica se muestra cómo dicho proceso se ha desarrollado durante más de sesenta años, a través de tres etapas caracterizadas por una educación ambiental superior centrada sucesivamente en los recursos naturales, el medio ambiente y la sustentabilidad de las sociedades. Para describir la situación actual de este proceso se presentan algunas de las experiencias más conocidas con respecto a la formación, la investigación, la extensión y la gestión ambiental, en 30 universidades de 13 países latinoamericanos (Ver Tabla No. 2).

Como señalan los autores, "desafortunadamente, este importante y muy rico proceso de ambientalización de la educación superior en América Latina y el Caribe está escasamente documentado a escala regional durante las últimas décadas". Tampoco existe ningún tipo de estadísticas para la región y, por lo tanto, solo se pueden hacer algunos cálculos muy generales. Extrapolando a partir de algunos estudios nacionales, "se puede esperar que actualmente el número de programas de formación ambiental en las universidades de la región sea de varios miles". Con seguridad, son cientos las instituciones de educación superior con algún grado de ambientalización importante. Estimaciones similares se pueden hacer para las acciones de investigación y extensión ambiental en las IES latinoamericanas y caribeñas: también "Ios 
centros de investigación son varios cientos; los grupos de investigación se cuentan por miles; los investigadores son algunas decenas de miles; y los proyectos de investigación - extensión son aún más numerosos" (SÁENZ \& BENAYAS, 2015, p. 206 y 212).

Las limitaciones en el conocimiento sobre el proceso de ambientalización de las instituciones de educación superior en América Latina y el Caribe son reconocidas desde hace un buen tiempo por las redes universitarias ambientales que integran ARIUSA. Para superarlas en sus ámbitos nacionales, algunas de ellas desarrollaron sus propios sistemas de indicadores o criterios para evaluar la incorporación de la dimensión ambiental en sus universidades. A su vez, desde la Coordinación de ARIUSA se promovió y apoyó la creación de un par de redes operativas que han desarrollado proyectos colaborativos de investigación para acordar y adoptar un sistema de indicadores comunes para las IES de la región (SÁENZ, 2015c, p. 141).

La más importante de estas iniciativas es la Red de Indicadores de Sostenibilidad en las Universidades (RISU), que se creó en junio de 2012 en el marco de la Segunda Jornada Iberoamericana de ARIUSA. Para cumplir con su objetivo central, en RISU se formuló el proyecto de investigación Definición de indicadores y evaluación de los compromisos con la sostenibilidad en Universidades Latinoamericanas. Este proyecto colaborativo internacional se desarrolló durante 2013 y 2014 para generar importantes resultados. El primero fue la construcción colectiva de un "conjunto de 114 indicadores distribuidos en 11 temáticas o dimensiones de la posible aplicación de la sostenibilidad en universidades" (BENAYAS et al, 2014, p. 18). Estos indicadores se utilizaron en una encuesta que fue respondida en línea por 65 universidades de 10 países latinoamericanos y caribeños (Ver Tabla No. 2).

Con el proyecto RISU se tuvo por primera vez una medición cuantitativa del compromiso con la sustentabilidad de las instituciones de educación superior en la región. Aunque no era representativa en el sentido estricto, "en relación con las, aproximadamente, 1.100 universidades reconocidas por los Ministerios de Educación de estos países, la muestra analizada supone cerca del $6 \%$ del total". Con respecto a los 11 ámbitos de indicadores que se utilizaron para el análisis, los resultados generales muestran que los mayores avances se refieren a la responsabilidad socioambiental, las políticas de sustentabilidad y la gestión de residuos. Por el contrario, las universidades presentaron un menor desarrollo en los ámbitos de la contratación responsable y las medidas para controlar el tráfico y la movilidad en la universidad (BENAYAS et al, 2014, p. 19 y 23).

\section{DiAgNósticoS NACIONALES Y REGIONAL}

Con base en la selección de un grupo de indicadores entre los acordados en el Proyecto RISU, desde 2014 se adelanta en ARIUSA una nueva serie de diagnósticos nacionales que permitirán llegar a un nuevo y más completo diagnóstico regional del estado actual de la institucionalización del compromiso ambiental de las universidades de América Latina y el Caribe.

La idea inicial para este trabajo surgió durante el desarrollo de una de las actividades preparatorias del III Encuentro Internacional de Rectores de UNIVERSIA, Rio 2014. Esta 
organización universitaria convocó durante el año 2013 a diferentes debates en internet sobre temas claves para las instituciones de educación superior en el mundo actual. Uno de estos debates académicos se planteó alrededor de la pregunta: ¿Le corresponde a la universidad un papel relevante en la estrategia de sostenibilidad socio-ambiental? La respuesta unánime de los participantes en este debate fue afirmativa y de este acuerdo inicial derivó una segunda pregunta: "¿Hasta qué punto las universidades de Iberoamérica han asumido su compromiso con la sostenibilidad ambiental?" (SÁENZ, 2013, p. 1).

Los esfuerzos por responder a este nuevo interrogante se convirtieron en el verdadero punto central del intercambio de ideas entre especialistas en el tema ambiental universitario, que se adelantó entre junio y septiembre de 2013. Muchos plantearon sus opiniones personales sobre el nivel de compromiso ambiental de las universidades iberoamericanas, pero nadie estuvo en condiciones de aportar datos que las respaldaran. A pesar de que se contaba con varios de los diagnósticos señalados en los apartados anteriores, ninguna de las afirmaciones se apoyaba en cifras sobre el grado de avance del proceso de ambientalización de las instituciones de educación superior en la región.

Para superar estas limitaciones en el conocimiento del proceso sobre el que se pretendía intervenir, se formularon algunas propuestas. El profesor Javier Benayas, de la Universidad Autónoma de Madrid, planteó la necesidad de "definir una agenda iberoamericana de compromisos concretos de las universidades hacia la sustentabilidad". Al apoyar esta iniciativa, el Coordinador de ARIUSA propuso elaborar "un plan de acción común" que permita, al mismo tiempo, conocer mejor y actuar más efectivamente sobre la situación actual de la sostenibilidad ambiental en las universidades. Como un primer componente necesario en este plan, señaló la necesidad de contar con un "diagnóstico sobre la sustentabilidad en las universidades de Iberoamérica" (SÁENZ, 2013, p. 2).

Estas propuestas sellevaron al I Foro Latinoamericano de Universidadesy Sostenibilidad, que tuvo lugar en Viña del Mar (Chile), en diciembre de 2013. Fueron aceptadas por los participantes en este evento como una sugerencia a los rectores universitarios en América Latina y el Caribe, en el sentido de "formular una Agenda para la Sostenibilidad en las Universidades de la región que oriente, a mediano plazo, el proceso de ambientalización y compromiso con la sostenibilidad de nuestras instituciones en los próximos años". Como uno de los puntos básicos de esta agenda se recomendó la "realización de un diagnóstico de la situación actual del proceso de ambientalización de las instituciones de educación superior a escala regional y en la mayoría de los países latinoamericanos y caribeños" (SÁENZ, 2015a, p. 127).

Las mismas iniciativas las presentó el Coordinador de ARIUSA en la Reunión de Puntos Focales de la Red de Formación Ambiental para América Latina y el Caribe (RFA-ALC), que se celebró en Bogotá a finales de febrero de 2014. En este tipo de reuniones participan los directores o representantes de las unidades de educación ambiental de los Ministerios de Ambiente en gran parte de los países latinoamericanos y caribeños. Como es habitual en los encuentros de los Puntos Focales de la RFA-ALC, previas a los Foros de Ministros, entre sus principales resultados se cuentan un conjunto de recomendaciones con relación a los temas de educación ambiental. 
Una de ellas se refería precisamente a "desarrollar un diagnóstico por país sobre la inclusión de consideraciones ambientales en las universidades" (MARTÍNEZ, 2014, p. 16).

Esta recomendación de la RFA-ALC fue acogida en el del XIX Foro de Ministros de Ambiente en América Latina y el Caribe, que tuvo lugar en la ciudad de Los Cabos (México) en marzo de 2014. Entre otros acuerdos, en su Decisión 2 sobre "Educación Ambiental para el Desarrollo Sostenible", los Ministros solicitaron a la Oficina Regional (ORPALC) del Programa de las Naciones Unidas para el Medio Ambiente (PNUMA) la formulación de un plan de trabajo de la RFA-ALC para el periodo 2014-2016. Como una de sus acciones, en este plan de trabajo se debía "desarrollar un diagnóstico por país sobre la inclusión de consideraciones ambientales en las universidades (en cuatro ámbitos que abarcan el currículum, la gestión institucional, la extensión y la investigación), con base, tanto en indicadores comunes, como diferenciados por país" (PNUMA, 2014, p. 3).

Consecuente con la responsabilidad que deriva de la formulación de la propuesta original, el Coordinador de ARIUSA se comprometió a promover el cumplimiento de dicha decisión en el mayor número posible de países de América Latina y el Caribe, con el apoyo directo de la Coordinación de la RFA-ALC. A esta tarea se han sumado varias de las Redes Universitarias Ambientales (RUAs) que integran la Alianza Iberoamericana y unidades de educación ambiental de los Ministerios de Ambiente de varios países de la región.

Los primeros en contar con estos diagnósticos nacionales fueron, en su orden: Perú, Colombia, Ecuador, México y Chile. Desde mediados de 2014 hasta comienzos del 2017, un total de 265 universidades en estos 5 países latinoamericanos respondieron las correspondientes encuestas nacionales (SAÉNZ, 2016, p.10), que tienen en común 25 indicadores básicos. Con excepción de Chile (por ser el más reciente), para cada uno de ellos también se tienen sus respectivos informes (CARDENAS, 2015), (SÁENZ et al, 2017), (TORRES \& CALDERÓN, 2015) (CECADESU \& ANUIES, 2016). En el primer semestre de 2017 se inició la encuesta en una nueva red de universidades de Nicaragua y una nueva fase del diagnóstico en Colombia con un formulario ampliado, que incluye las mismas 25 preguntas, compartidos por todas las encuestas. Para el segundo semestre está anunciada la ejecución del diagnóstico en el Estado de Rio Grande do Sul (Brasil), así como en Argentina. Se esperan anuncios similares en otros países.

Es probable que esta dinámica de trabajo colaborativo entre las redes en ARIUSA y los Ministerios de Ambiente se acelere y amplíe significativamente. Con los resultados de un alto número de encuestas nacionales se podrá tener un buen diagnóstico de la "ambientalización de las instituciones de educación superior a escala regional", como se propuso desde 2013 en Viña del Mar. Sin embargo, lograr la participación de la mayoría de los países latinoamericanos y caribeños es una tarea que requerirá varios años más de esfuerzo sistemático y permanente.

\section{BALANCE GENERAL DE LOS DIAGNÓSTICOS}

Según se ha visto, es reducido el número de estudios que se pueden considerar como diagnósticos de escala regional o subregional sobre la ambientalización de las instituciones de educación superior en América Latina y el Caribe. Desde que se iniciaron en 1977, fueron 
apenas 4 en las primeras tres décadas y otros 3 en los últimos años. Desde 2014 se adelanta una serie de encuestas nacionales, con 25 indicadores comunes, que permitirán tener un gran diagnóstico regional.

De los 8 estudios registrados, 7 son de escala regional y solo 1 de ámbito subregional. Este último es una auditoría ambiental a un pequeño grupo de universidades caribeñas en países de habla inglesa. Una síntesis de todos estos diagnósticos se presenta en la Tabla No.1.

Tabla 1 - Diagnósticos regionales y subregional, en América Latina y el Caribe, sobre la incorporación de la dimensión ambiental en las instituciones de educación superior

\begin{tabular}{|c|l|l|l|l|}
\hline Año & \multicolumn{1}{|c|}{ Diagnóstico Regional o Subregional } & Países & IES & Fuente \\
\hline 1977 & $\begin{array}{l}\text { Panorama de los Estudios Superiores Medioambientales en } \\
\text { América Latina }\end{array}$ & 12 & 73 & $(1)$ \\
\hline 1984 & $\begin{array}{l}\text { Diagnóstico de la Incorporación de la Dimensión Ambiental en } \\
\text { los Estudios Superiores en América Latina y el Caribe }\end{array}$ & 17 & 166 & $(2)$ \\
\hline 2000 & $\begin{array}{l}\text { Informes Nacionales. Memorias del III Congreso Iberoamericano } \\
\text { de Educación Ambiental }\end{array}$ & 12 & $\begin{array}{l}\text { Sin } \\
\text { dato }\end{array}$ & $(3)$ \\
\hline 2004 & $\begin{array}{l}\text { Educación Ambiental Superior en América Latina. Retos } \\
\text { Epistemológicos y Curriculares }\end{array}$ & 13 & 64 & $(4)$ \\
\hline 2010 & $\begin{array}{l}\text { Report of the Audit - Mainstreaming Environment and } \\
\text { Sustainability in Caribbean Universities }\end{array}$ & 4 & 6 & $(5)$ \\
\hline 2012 & $\begin{array}{l}\text { Higher Education, Environment and Sustainability in Latin } \\
\text { America and the Caribbean }\end{array}$ & 13 & 30 & $(6)$ \\
\hline 2014 & $\begin{array}{l}\text { Definición de Indicadores y Evaluación de los Compromisos con } \\
\text { la Sostenibilidad en Universidades Latinoamericanas }\end{array}$ & 10 & 65 & $(7)$ \\
\hline 2014 & $\begin{array}{l}\text { Diagnósticos Nacionales sobre la Institucionalización del } \\
\text { Compromiso Ambiental en las Universidades de ALC }\end{array}$ & 5 & 265 & $(8)$ \\
\hline
\end{tabular}

Fuentes: 1 (CIFCA, 1978); 2 (PNUMA, 1988); 3 (ÁLVAREZ, 2002); 4 (ESCHENHAGEN, 2009); 5 (UWI \& UNEP, 2011); 6 (SÁENZ \& BENAYAS, 2012); 7 (BENAYAS et al, 2014); 8 (CARDENAS, 2015), (SÁENZ et al, 2017), (TORRES \& CALDERÓN, 2015), (CECADESU \& ANUIES, 2016).

Aunque el número total es pequeño, es de resaltar que América Latina y el Caribe es la única región en el mundo que cuenta con este tipo de estudios. Hasta donde conoce el autor, no se tienen diagnósticos similares en regiones como América del Norte, Europa, África o Asia.

Ninguno de los estudios regionales ha reportado información sobre universidades de todos los países de la región. Hasta el momento, el que mayor número comprendió fue el Diagnóstico de la Incorporación de la Dimensión Ambiental en los Estudios Superiores en América Latina y el Caribe, con un total de 17 países, la mayoría de ellos latinoamericanos. Por su parte, el Report of the Audit - Mainstreaming Environment and Sustainability in Caribbean Universities incluyó solo 4 países de esta subregión. (Ver Tabla No. 2).

Si se consideran los 8 estudios en su conjunto, se cuenta con información sobre universidades y otras instituciones de educación superior en un total de 21 países de la región. Como referencia, 
se pueden tomar las 33 naciones que son miembros de la Comunidad de Estados Latinoamericanos y Caribeños (CELAC). Por lo tanto, los datos disponibles corresponden al 63,6\% de estos países.

Tabla 2 - Países con información en los diagnósticos regionales y subregional sobre la

incorporación de la dimensión ambiental en las instituciones de educación superior de ALC

\begin{tabular}{|c|c|c|c|c|c|c|c|c|c|c|}
\hline \multirow[b]{2}{*}{ No. } & \multirow[b]{2}{*}{ PAÍs } & \multicolumn{4}{|c|}{ Primeros diagnósticos } & \multicolumn{4}{|c|}{ Diagnósticos recientes } & \multirow[b]{2}{*}{ TOTALES } \\
\hline & & $\begin{array}{c}1977 \\
(1)\end{array}$ & $\begin{array}{c}1984 \\
(2)\end{array}$ & $\begin{array}{c}2000 \\
(3)\end{array}$ & $\begin{array}{c}2004 \\
(4)\end{array}$ & $\begin{array}{c}2010 \\
(5)\end{array}$ & $\begin{array}{c}2012 \\
(6)\end{array}$ & $\begin{array}{c}2014 \\
(7)\end{array}$ & \begin{tabular}{|c|}
2014 \\
2017 \\
$(8)$ \\
\end{tabular} & \\
\hline 1 & Argentina & $\sqrt{ }$ & $\sqrt{ }$ & $\sqrt{ }$ & $\sqrt{ }$ & & $\sqrt{ }$ & $\sqrt{ }$ & & 6 \\
\hline 2 & Barbados & & & & & $\sqrt{ }$ & & & & 1 \\
\hline 3 & Belice & & & & & $\sqrt{ }$ & & & & 1 \\
\hline 4 & Bolivia & $\sqrt{ }$ & $\sqrt{ }$ & & $\sqrt{ }$ & & $\sqrt{ }$ & & & 4 \\
\hline 5 & Brasil & & $\sqrt{ }$ & $\sqrt{ }$ & $\sqrt{ }$ & & $\sqrt{ }$ & $\sqrt{ }$ & & 5 \\
\hline 6 & Colombia & $\sqrt{ }$ & $\sqrt{ }$ & $\sqrt{ }$ & $\sqrt{ }$ & & $\sqrt{ }$ & $\sqrt{ }$ & $\sqrt{ }$ & 7 \\
\hline 7 & Costa Rica & $\sqrt{ }$ & $\sqrt{ }$ & $\sqrt{ }$ & $\sqrt{ }$ & & $\sqrt{ }$ & $\sqrt{ }$ & & 6 \\
\hline 8 & Chile & $\sqrt{ }$ & $\sqrt{ }$ & $\sqrt{ }$ & $\sqrt{ }$ & & $\sqrt{ }$ & $\sqrt{ }$ & $\sqrt{ }$ & 7 \\
\hline 9 & Cuba & & $\sqrt{ }$ & $\sqrt{ }$ & $\sqrt{ }$ & & & & & 3 \\
\hline 10 & Ecuador & $\sqrt{ }$ & $\sqrt{ }$ & & $\sqrt{ }$ & & $\sqrt{ }$ & & $\sqrt{ }$ & 5 \\
\hline 11 & Guatemala & $\sqrt{ }$ & $\sqrt{ }$ & & $\sqrt{ }$ & & $\sqrt{ }$ & $\sqrt{ }$ & & 5 \\
\hline 12 & Haití & & $\sqrt{ }$ & & & & & & & 1 \\
\hline 13 & Jamaica & & & & & $\sqrt{ }$ & & & & 1 \\
\hline 14 & México & $\sqrt{ }$ & $\sqrt{ }$ & $\sqrt{ }$ & $\sqrt{ }$ & & $\sqrt{ }$ & $\sqrt{ }$ & $\sqrt{ }$ & 7 \\
\hline 15 & Panamá & & $\sqrt{ }$ & & $\sqrt{ }$ & & $\sqrt{ }$ & & & 3 \\
\hline 16 & Paraguay & $\sqrt{ }$ & $\sqrt{ }$ & & & & $\sqrt{ }$ & & & 3 \\
\hline 17 & Perú & $\sqrt{ }$ & $\sqrt{ }$ & $\sqrt{ }$ & $\sqrt{ }$ & & $\sqrt{ }$ & $\sqrt{ }$ & $\sqrt{ }$ & 7 \\
\hline 18 & $\begin{array}{l}\text { República } \\
\text { Dominicana }\end{array}$ & & $\sqrt{ }$ & $\sqrt{ }$ & & & $\sqrt{ }$ & $\sqrt{ }$ & & 4 \\
\hline 19 & Trinidad y Tobago & & & & & $\sqrt{ }$ & & & & 1 \\
\hline 20 & Uruguay & $\sqrt{ }$ & $\sqrt{ }$ & & & & & & & 2 \\
\hline 21 & Venezuela & $\sqrt{ }$ & $\sqrt{ }$ & $\sqrt{ }$ & $\sqrt{ }$ & & & $\sqrt{ }$ & & 5 \\
\hline & TOTALES & 12 & 17 & 10 & 13 & 4 & 13 & 10 & 5 & \\
\hline
\end{tabular}

Fuentes: 1 (CIFCA, 1978); 2 (PNUMA, 1988); 3 (ÁLVAREZ, 2002); 4 (ESCHENHAGEN, 2009); 5 (UWI \& UNEP, 2011); 6 (SÁENZ \& BENAYAS, 2012); 7 (BENAYAS et al, 2014); 8 (CARDENAS, 2015), (SÁENZ et al, 2017), (TORRES \& CALDERÓN, 2015), (CECADESU \& ANUIES, 2016).

Como se puede apreciar en la Tabla No. 2, la participación de estos 21 países en los diferentes diagnósticos regionales y subregional ha sido desigual. Algunos países, como Colombia, Chile, México y Perú han participado en todos los 7 diagnósticos de escala regional que se han hecho. Otros, como Haití y los 4 países de habla inglesa incluidos en el reporte subregional, solo han participado en uno de estos estudios.

Otro aspecto importante a considerar es el número de universidades y otras instituciones de educación superior que han aportado o las que se refiere la información en los mencionados diagnósticos (Ver Tabla No. 1). Durante mucho tiempo, el estudio más amplio en este sentido fue el diagnóstico realizado por el PNUMA en 1984, que logró recopilar información de 166 
universidades, en su gran mayoría latinoamericanas. En contraste, la auditoría subregional incluyó apenas 6 universidades caribeñas de habla inglesa.

Los diagnósticos nacionales que se están desarrollando desde 2014 han aportado información de un número bastante más alto de instituciones de educación superior. En los primeros 5 países donde se ha aplicado la encuesta común, el total de IES llega a 265. Es muy probable que en los próximos años se pueda tener datos comparables para más de 400 universidades de América Latina y el Caribe.

Un primer problema que presentan los diagnósticos regionales es su falta de articulación. Ninguno de estos estudios se ha propuesto darle continuidad a alguno de los anteriores. Aún peor, la mayoría de los autores de estos trabajos ignoran los otros. Otro problema de los 8 estudios regionales y subregional reseñados es que utilizan diferentes enfoques y procedimientos de investigación, que impiden hacer comparaciones cuantitativas válidas entre sus resultados.

Esta situación hace muy difícil tener un buen conocimiento de las diferentes etapas y avances en el proceso de institucionalización del compromiso ambiental en las universidades latinoamericanasy caribeñas. Para lograrlo de manera limitada, se puede intentar una descripción cualitativa general del proceso de ambientalización de las universidades latinoamericanas y caribeñas, a partir de la información que aportan los diagnósticos reseñados.

Los resultados de un ejercicio de este tipo se presentaron en la ponencia Panorama de la Sustentabilidad en las Universidades de América Latina y el Caribe (SÁENZ, 2014), que se realizó por solicitud de los organizadores del IV Seminário Sustentabilidade na Universidade: desafios à ambientalização nas Instituições de Ensino Superior no Brasil. El primero de los objetivos de este evento era verificar el estado del arte de la incorporación de la temática ambiental y de la sustentabilidad socioambiental en las instituciones de enseñanza superior. En particular interesaba conocer el panorama de la sustentabilidad en las IES latinoamericanas.

Con respecto al "estado del arte actual", dicha ponencia concluyó que el conocimiento sobre el proceso de ambientalización de las universidades latinoamericanas y caribeñas es muy limitado. "Muchos aspectos importantes de este proceso todavía no se conocen. Todos los estudios se mueven entre registros o reflexiones sobre experiencias en universidades particulares y reportes sobre el estado o las grandes tendencias a escala regional y nacional". A su vez, en el "panorama general" del objeto de estudio se observa que "en la gran mayoría de los países latinoamericanos son cada vez más numerosas las universidades con algún grado de ambientalización, pero no se tiene certeza sobre el nivel de desarrollo cuantitativo que se han alcanzado" (SÁENZ, 2014, pp. 34 y35).

\section{De los diAgNósticos AL OBSERVATORIO}

Este balance general de los diagnósticos regionales y subregional también es válido para los estudios nacionales que se reseñaron en otro artículo (SAÉNZ, 2015b), pues presentan deficiencias similares desde el punto de vista metodológico y conceptual. Por esta razón, 
no se ha logrado aún cumplir con una aspiración expresada desde hace tres décadas por la comunidad académica y científica que promueve y estudia la institucionalización del compromiso ambiental de las universidades latinoamericanas y caribeñas: contar con un registro sistemático y permanente sobre las actividades de incorporación de la dimensión ambiental en la educación superior.

Esta necesidad fue planteada a finales de 1985 por los participantes en el Seminario de Bogotá (Colombia) en el que se presentaron los resultados del diagnóstico realizado por el PNUMA. Entre las recomendaciones que se formularon en este evento sobre Universidad y Medio Ambiente en América Latina y el Caribe se destaca la de "continuar la recolección y difusión de información sobre Programas Ambientales Universitarios en la región, para completar el diagnóstico presentado en este seminario". Adicionalmente, se propuso "el establecimiento, a mediano plazo, de un sistema de recopilación, análisis y difusión de información relativo a los programas ambientales universitarios a nivel regional" (UNESCO, PNUMA \& ICFES, 1988, pp.92 y 93).

Desafortunadamente, estas recomendaciones no fueron asumidas por la Red de Formación Ambiental para América Latina y el Caribe (RFA-ALC) ni por alguna otra institución internacional que pudiera ponerlas en práctica. Más de 30 años después no se cuenta con un sistema de información que permita hacer el seguimiento de los avances cuantitativos del proceso de incorporación de la dimensión ambiental en las instituciones de educación superior en la región, ni con un sistema de evaluación que pueda dar cuenta de sus aspectos cualitativos en general.

Como ya se informó en uno de los apartados anteriores, una propuesta en la misma dirección se presentó en diciembre de 2013, en el I Foro Latinoamericano de Universidades y Sostenibilidad. Como una sugerencia a los rectores universitarios de la región, se propuso formular una "Agenda para la Sostenibilidad en las Universidades" que debería incluir como mínimo: a) Un sistema de indicadores de la sostenibilidad en las IES; b) Un diagnóstico regional con los respectivos diagnósticos nacionales; y c) el "establecimiento de un Sistema de Información que permita hacer un seguimiento permanente a los avances del compromiso de las universidades con la sustentabilidad" (SAENZ, 2015a, p. 128).

Consecuente con el principio de poner en práctica lo que propone, desde la Coordinación de ARIUSA se viene promoviendo la implementación de dicha "Agenda para la Sostenibilidad en las Universidades" de manera sistemática y rigurosa, en un proceso de trabajo colectivo que ya lleva cuatro años y debe continuar por muchos más. La "construcción de un sistema de indicadores de la sostenibilidad en las Universidades" de la región se logró a través del Proyecto RISU, con la selección consensuada de un conjunto de 114 indicadores y su puesta a prueba en una encuesta que respondieron 65 universidades latinoamericanas. El objetivo de contar con "un diagnóstico de la situación actual del proceso de ambientalización de las instituciones de educación superior a escala regional y en la mayoría de los países latinoamericanos y caribeños" (SAENZ, 2015a, p. 127) también se está implementando a través de la serie de diagnósticos nacionales que terminarán con un diagnóstico regional basado en la información proporcionada por un número muy alto de universidades y otras IES. 
Con el cumplimiento progresivo de estos dos primeros objetivos se están creando las condiciones para lograr el tercero: construir y mantener actualizado un sistema de información permanente sobre el proceso de ambientalización y compromiso con la sustentabilidad de las universidades de la región. Del conjunto de indicadores de RISU se seleccionaron 25 indicadores básicos para elaborar una encuesta común que se está aplicando en un número creciente de países latinoamericanos. Ya se ha indicado que, hasta mayo de 2017, han respondido los respectivos formularios nacionales un total de 265 universidades y otras IES de Perú, Colombia, Ecuador, México y Chile. Además, se están iniciando o preparando los respectivos diagnósticos nacionales o estatales en Nicaragua, Argentina y el Estado de Rio Grande do Sul (Brasil).

Los indicadores acordados y la gran cantidad de información que se está recopilando de manera cuidadosa y sistemática constituyen la base necesaria para el propuesto sistema de información. Pero se puede dar un paso adicional y sumar un nuevo elemento a la "Agenda para la Sostenibilidad en las Universidades" planteada por ARIUSA. Para que el sistema de información permita "hacer un seguimiento permanente a los avances del compromiso de las universidades con la sustentabilidad" es necesario construir un observatorio, como muchos otros que se ocupan de hacer el seguimiento y evaluación permanente de diferentes procesos sociales, económicos y políticos de interés.

En este sentido se propone crear en el mediano plazo un Observatorio de la Sustentabilidad en la Educación Superior (OSES). Este observatorio se encargará de recopilar información y analizar de manera continua el proceso por el cual las instituciones de educación superior en América Latina y el Caribe incorporan los temas de ambiente y sustentabilidad en sus funciones sustantivas de formación, investigación y extensión, así como en sus acciones de gobierno y gestión institucional (SAENZ, 2015d). Como base inicial para la construcción del OSES se pueden tomar los resultados de los diagnósticos nacionales que ya se tienen y de los nuevos que se realicen en los próximos años.

Otro procedimiento para hacer un seguimiento permanente de los avances de las instituciones de educación superior en su compromiso con la sustentabilidad será la revisión continua de sus sitios web, en los que informan sobre sus acciones en materia de ambiente. Así se está haciendo en Colombia desde 2015, en la segunda fase del diagnóstico nacional que se adelanta en este país. Desde el observatorio se promoverá que todas las universidades o instituciones similares en la región reporten en sus páginas de web sus actividades, proyectos y programas de carácter ambiental.

Una tercera y muy importante fuente para obtener información sobre el compromiso de las universidades con la sustentabilidad son los reportes o informes ambientales que algunas instituciones de educación superior están comenzando a presentar con alguna periodicidad. Esta práctica es más común en otras regiones del mundo; en América Latina y el Caribe está comenzando a adoptarse y será promovida aún más desde el OSES.

Para la divulgación de los resultados de los análisis, el OSES contará con su propio sitio web y con diversas publicaciones periódicas. A través de estos y otros medios se informará a los interesados en el tema, especialmente a las instituciones y redes ambientales que promueven la institucionalización del compromiso ambiental en las universidades de la región. 
En particular, para las redes en ARIUSA el Observatorio de la Sustentabilidad en Educación Superior será un instrumento de gran utilidad en la evaluación de los resultados de su trabajo. No obstante que se cuenta con la información proporcionada por varias decenas de diagnósticos nacionales, subregional y regionales, ninguna de estas redes puede ahora conocer los avances que han tenido las universidades asociadas en su compromiso con el ambiente y la sustentabilidad. Con la información del OSES estarán en condiciones de hacerlo.

\section{REFERENCIAS}

ÁlVAREZ, A. et al. Informes Nacionales. En: III Congreso Iberoamericano de Educación Ambiental. Memorias. Caracas: Ministerio de Ambiente y Recursos Naturales y Programa de las Naciones para el Medio Ambiente. pp. 645-969. 2002.

BENAYAS, J. et al. Resumen Ejecutivo Proyecto RISU. Definición de indicadores para la evaluación de las políticas de sustentabilidad en Universidades Latinoamericanas. Madrid: Universidad Autónoma de Madrid y Alianza de Redes Iberoamericanas de Universidades por la Sustentabilidad y el Ambiente. 52 pp. 2014.

CÁRDENAS, J. M. Evaluación de la Incorporación de la Dimensión Ambiental en las Universidades del Perú. En: Revista Iberoamericana Universitaria en Ambiente, Sociedad y Sustentabilidad (AMBIENS). Vol. 1 No. 2. pp. 161-178. Julio - Diciembre. 2015.

CECADESU \& ANUIES. Diagnóstico sobre la incorporación de la perspectiva ambiental en las funciones sustantivas de la Instituciones de Educación Superior en México. México: Centro de Educación y Capacitación para el Desarrollo Sustentable y Asociación Nacional de Universidades e Instituciones de Educación Superior. 42 pp. Enero de 2016.

CIFCA. Panorama de los Estudios Superiores Medioambientales en América Latina. En: La Formación Ambiental en América Latina. Cuadernos del CIFCA No. 8. Madrid: Centro Internacional de Formación en Ciencias Ambientales. pp. 55-139. 1978.

CURIEL, A. (Compilador). Educación Ambiental y Universidad. Memorias del primer Congreso Iberoamericano de Educación Ambiental. Guadalajara: Universidad de Guadalajara. Colección Universidad y Ambiente. 445 pp. 1993.

ESCHENHAGEN, M. L. Educación Ambiental Superior en América Latina. Retos Epistemológicos y Curriculares. Bogotá: Red Colombiana de Formación Ambiental. Biblioteca Universidad y Ambiente. Vol. IV. 259 pp. 2009.

GUNi. Higher Education in the World 4. Higher Education's Commitment to Sustainability: from Understanding to Action. Barcelona: Global University Network for Innovation (GUNi) and Palgrave MacMillan. 341 pp. 2012.

KO, N., ABE, O. Sustainability and Higher Education in Asia and Pacific. En: GUNi. Higher Education in the World 4. Higher Education's Commitment to Sustainability: from Understanding to Action. Barcelona: Global University Network for Innovation (GUNi) and Palgrave MacMillan. pp. 84-96. 2012.

LEFF, E. La formación ambiental superior en la perspectiva de la Cumbre de la Tierra y de la Agenda 21. En Curiel, A. (Compilador). Educación Ambiental y Universidad. Memorias del primer Congreso Iberoamericano de Educación Ambiental. Guadalajara: Universidad de Guadalajara. Colección Universidad y Ambiente. pp. 74-92. 1993. 
LOTZ-SISITKA, L. The Event of Modern Sustainable Development and Universities in Africa. En: GUNi. Higher Education in the World 4. Higher Education's Commitment to Sustainability: from Understanding to Action. Barcelona: Global University Network for Innovation (GUNi) and Palgrave MacMillan. pp. 41-57. 2012.

MARTINEZ, I. Memoria de la Reunión de Puntos Focales de la Red de Formación Ambiental para América Latina y el Caribe. Panamá: Programa de las Naciones Unidas para el Medio Ambiente, Red de Formación Ambiental para América Latina y el Caribe. 25 pp. 2014.

NAVARRRO, E. Comparative Analysis of the Audit Studies of RISU and MESCA. En: Revista Iberoamericana Universitaria en Ambiente, Sociedad y Sustentabilidad (AMBIENS). Vol. 1 No. 1 Junio - Diciembre. pp. 192-257. 2015.

PNUMA. Decisión del XIX Foro de Ministros de Medio Ambiente para América Latina y el Caribe sobre Educación Ambiental para el Desarrollo Sostenible. Programa de las Naciones Unidas para el Medio Ambiente. 4 pp. 2014.

RIKERS, J.; SNOO, G.R. de; van DAM-MIERAS, M. Higher Education and Sustainability in Europe. En: GUNi. Higher Education in the World 4. Higher Education's Commitment to Sustainability: from Understanding to Action. Barcelona: Global University Network for Innovation (GUNi) and Palgrave MacMillan. pp. 113127. 2012.

SÁENZ, O. La Formación Ambiental Superior. 1948-1991. Bogotá: Universidad de Ciencias Aplicadas y Ambientales y Red Colombiana de Formación Ambiental. 248 p. 2012.

SÁENZ, O. Debate sobre la Sostenibilidad Ambiental en las Universidades. Resumen. III Encuentro de Rectores UNIVERSIA Rio 2014. Bogotá: Universidad de Ciencias Aplicadas y Ambientales (U.D.C.A). 2 pp. 2013.

SÁENZ, O. Panorama de la Sustentabilidad en las Universidades de América Latina y el Caribe. Em: RUSCHEINSKY, A. et al (Organizadores). Ambientalização nas Instituições de Educação Superior no Brasil: Caminhos Trilhados, Desafios e Possibilidades. São Paulo: Universidade de São Paulo. pp. 23-38. 2014.

SAENZ, O. (Editor). Universidades y Sostenibilidad en América Latina y el Caribe. Bogotá: Universidad de Ciencias Aplicadas y Ambientales y Red Colombiana de Formación Ambiental. 130 pp. 2015 a.

SÁENZ, O. Diagnósticos Nacionales sobre la Inclusión de Consideraciones Ambientales en las Universidades de América Latina y El Caribe. En: Revista Iberoamericana Universitaria en Ambiente, Sociedad y Sustentabilidad (AMBIENS). Vol. 1 No. 1 Enero - Junio. pp. 13-36. 2015 b.

SÁENZ, O. Trayectoria y resultados del Proyecto RISU en el contexto de ARIUSA. En: Contrapontos. Vol. 15 No. 2 Mayo - Agosto. pp. 137-164. 2015c.

SAENZ, O. Propuesta para la Creación del Observatorio de la Sustentabilidad en la Educación Superior. Documento de trabajo. Bogotá: Universidad de Ciencias Aplicadas y Ambientales. 7 pp. 2015 d.

SÁENZ, O. et al. Institucionalización del Compromiso Ambiental de las Universidades Colombianas. Bogotá: Equipo Universidad, Ambiente y Sustentabilidad (UAS). Artículo próximo a publicar en la Revista Civilizar. 2017.

SÁENZ, O.; BENAYAS, J. Higher Education, Environment and Sustainability in Latin America and the Caribbean. En: GUNi. Higher Education in the World 4. Higher Education's Commitment to Sustainability: from Understanding to Action. Barcelona: Global University Network for Innovation (GUNi) and Palgrave MacMillan. pp. 161-176. 2012. 
SÁENZ, O.; BENAYAS, J. Ambiente y Sustentabilidad en las Instituciones de Educación Superior en América Latina y el Caribe. En: Revista Iberoamericana Universitaria en Ambiente, Sociedad y Sustentabilidad (AMBIENS). Vol. 1 No. 1 Junio - Diciembre. pp. 192-224. 2015.

SALAMÉ, R. Higher Education Commitment to Sustainability in the Arab States. En: GUNi. Higher Education in the World 4. Higher Education's Commitment to Sustainability: from Understanding to Action. Barcelona: Global University Network for Innovation (GUNi) and Palgrave MacMillan. pp. 69-75. 2012.

TORRES, R.S.; CALDERÓN, E. Diagnóstico sobre la Inclusión de Consideraciones Ambientales y de Sostenibilidad en las Universidades del Ecuador - Primera fase. En: Revista Iberoamericana Universitaria en Ambiente, Sociedad y Sustentabilidad (AMBIENS). Vol. 1 No. 2. pp. 110-119. Julio - Diciembre. 2015.

UNESCO; PNUMA; ICFES. Diagnóstico de la Incorporación de la Dimensión Ambiental en los Estudios Superiores en América Latina y el Caribe. En: Universidad y Medio Ambiente en América Latina. Seminario de Bogotá. Realizado del 28 de octubre al 1 de noviembre de 1985. México. D.F.: Organización de las Naciones Unidas para la Educación, la Ciencia y la Cultura, Programa de las Naciones Unidas para el Medio Ambiente e Instituto Colombiano para el Fomento de la Educación Superior. pp. 15-27 pp. 1988.

UWI; UNEP. Report of the Audit - Mainstreaming Environment and Sustainability in Caribbean Universities (MESCA). Kingston: University of West Indies and United Nations Environment Programme. 189 pp. 2011.

WRIGTH, T.; ELLIOT, H. Canada and US Regional Report. En: GUNi. Higher Education in the World 4. Higher Education's Commitment to Sustainability: from Understanding to Action. Barcelona: Global University Network for Innovation (GUNi) and Palgrave MacMillan. pp. 140-153. 2012.

Artigo recebido em: 09/05/2017

Aprovado em: 03/08/2017

\section{CONTATO PARA CORRESPONDÊNCIA:}

Orlando Sáenz.E-mail: osaenz@udca.edu.co 